\title{
AN INTERACTIVE DRUG SUPPLY CHAIN TRACKING SYSTEM USING BLOCKCHAIN 2.0
}

\author{
U. Padmavathi ${ }^{1}$ \\ Department of Computer Science \& Engineering, \\ National Institute of Technology Puducherry, Karaikal, Puducherry, India. \\ udayarajepadma@gmail.com \\ Narendran Rajagopalan ${ }^{2}$ \\ Department of Computer Science \& Engineering, \\ National Institute of Technology Puducherry, Karaikal, Puducherry, India. \\ narendran@nitpy.ac.in
}

\begin{abstract}
The modern pharmaceutical supply chain is a complex process in which researches is carried out to produce drugs and according to the research made the drugs is manufactured and these drugs are distributed from the manufacturer to the pharmacies. This process involves a number of stakeholders from manufacturer, wholesaler, distributor, pharmacies and finally end-users, the patients. At each stage, inventory must be managed based on demand in pharmaceutical products. Inventory management system is vital because an excess of inventory could cost the pharmacy money and it is more complex because it needs to track the lot numbers and expiration dates of medicines. Pharmaceutical supply chain suffers from various issues such as drug shortages, temperature control of drugs, lack of visibility in shipment and storage, inventory management, lack of coordination and the most important one drug counterfeiting. The principal regulatory bodies responsible for drug quality try to solve these issues by various ways. But they are very much unregulated, expensive and fragmented. The blossoming technology, Blockchain by its inherent properties such as immutability, transparency, and distributed nature could solve the problems of pharmaceutical supply chain. This paper starts with an introduction about Drug supply chain and their problems. It elucidates how Blockchain Technology could come to rescue pharmaceutical supply chain. This paper proposes a novel drug supply chain management system along with inventory management based on Blockchain 2.0 specifically Hyperledger Fabric. The proposed system records all transactions using Blockchain, thus helping in tracking the drug along its supply chain as well as solving the issues associated with drug supply chain in an efficient manner. It also measures performance of the proposed system using the Benchmark tool called Hyperledger caliper. The system proves its efficiency in terms of success rate, throughput and transaction latency.
\end{abstract}

Keywords: Blockchain, Counterfeit Drugs, Drug Supply Chain, Hyperledger Fabric, Hyperledger Caliper.

\section{Introduction}

In the current era, new drugs are introduced into the market daily as there is a rapid increase in the number of diseases among human beings. These drugs help the patient to recover from illness and sometimes they have some exacerbating effect on humans. The adverse outcome of drugs is mainly due to drug counterfeiting. This is because the lucrative drug changes hands many times along the supply chain which provides an opportunity for falsified drugs to enter into the market easily. Identifying these counterfeit drugs is complex and expensive. Even the physician who prescribes the drugs is not able to find the difference between the licit and illicit ones. The counterfeited drugs have profound and devastating effect on human beings.[1]

The term "counterfeit drug" refers to pharmaceutical item that are fraudulently mislabeled with respect to source and/or identity. It could also be defined as a product with wrong ingredient or a product with inactive ingredient or a product with active ingredient at high dosage. According to a research report by World Health Organization (WHO), an estimated one in 10 medical products circulating in low-and-middle income countries is either falsified or substandard [2].

The Indian Pharma industry which grows steadily has a huge place for spurious and counterfeit drugs. It is expected that the Pharma market in India would grow up to $\$ 55$ billion by 2020 [3]. Another report by WHO states that India is the third largest producer of generic drugs in the world and it plays a vital role in counterfeit pharmaceutical manufacturing. This counterfeiting of drugs is done keeping in mind that it is difficult to detect 
counterfeiting, lack of public awareness, leakage points in the supply chain and it needs low investment. Counterfeited drugs also find its way into the market when there is a shortage of specific drug that is on high demand[4]. Falsified drug producers take this opportunity and rush to fill the gap between supply and demand. Counterfeit medicines could result in failure to treat diseases, helps in the evolution of bugs that cause diseases and sometimes, it may be poisonous which result in fatality. In case of low-income countries, antibiotics and anti-malarias are the most commonly counterfeited medicines. Fraudsters are very much interested in manufacturing an exact copy of expensive prescription drugs such as drugs used in the treatment of AIDS, cancer. They do not bother about the quality and effectiveness of the counterfeited drugs.

Another major problem faced by the Pharmaceutical Supply chain system is the management of inventory at each stakeholder's point. Inventory management is vital in order to improve operational efficiency as well as to reduce costs, and wastage of medicines [5]. This process involves tracking of inventories and preparing the inventory based on demand, tracking expiration dates of medicines and so on. The inventory must be properly maintained which would otherwise result in wastage of medications, wastage of money on excess medications, a cut in the marginal profit of the company.

Other than drug counterfeiting and lack of inventory management, the Pharmaceutical supply chain due to its convoluted nature, suffers from the following issues.

1. Adverse Drug Reaction on patients

2. Drug shortages

3. Contaminated drug manufacturing

4. Improper Cold chain management

5. Lack of visibility in shipment and storage

\subsection{Blockchain}

Blockchain, a distributed ledger technology could simply be defined as chain of blocks that are linked to each other. Each block contains a record of transactions along with block header. The block header comprises current block hash, previous block hash, difficulty, timestamp and Nonce. The concept of blockchain has its birth with Bitcoin, the digital currency founded by Satoshi Nakamoto in the year 2008 as an alternate for physical currency[6]. The following Figure 1 illustrates Blockchain Structure.

\begin{tabular}{lll} 
Block N-1 Block N Block N+1 & \\
\hline
\end{tabular}

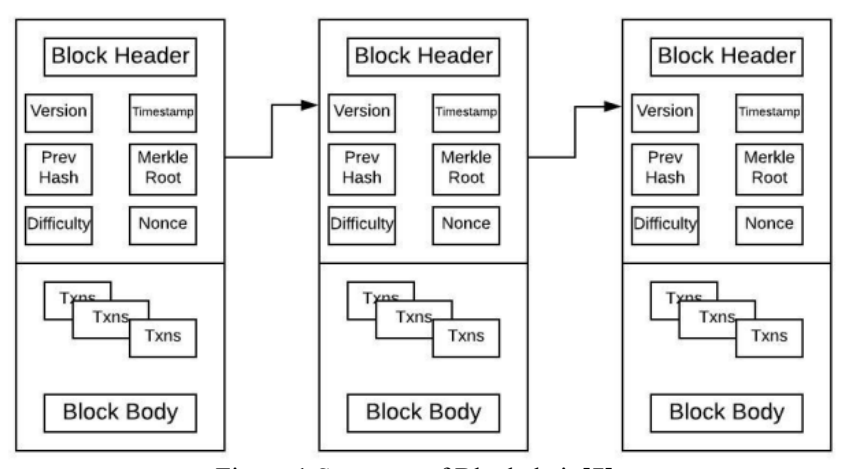

Figure.1 Structure of Blockchain[7]

The blocks in the blockchain are linked to the next block using the hash value. This provides immutability property which is a distinguishing feature of blockchain. Bitcoin blockchain achieves consensus using Proof of Work (PoW), a resource consuming mechanism in which the miners solve cryptographic puzzles in order to achieve their difficulty target. But this Bitcoin blockchain suffers from scalability problem and also it could not be applied to any other application. This gives birth to blockchain 2.0 which makes use of smart contracts. Smart contracts are computer codes that contain the actual logic of the business process. These smart contracts are executed automatically and help businesses to make use of blockchain for their applications. Blockchain may be public, private or consortium. A public Blockchain is the one in which anyone in the world can join and have access to this network at any time whereas a private blockchain is the one in which the single entity or organization that owns the blockchain has the full control over it. In case of consortium blockchain, a consortium of organizations has the control over the blockchain. This is also called as federated blockchain.

Drug Supply chain involves a number of stakeholders along its way which rises the need to make use of consortium blockchain so that every stakeholder involved in the system could have their control over the blockchain network. The authors found that Hyperledger Fabric Blockchain best suits this application. Hyperledger Fabric, one of the umbrella projects of IBM, is an open-source, permissioned blockchain. Hyperledger Fabric blockchain differs from other blockchain in many ways. It makes use of execute-ordervalidate mechanism whereas other blockchain networks use order-execute logic. This helps Hyperledger Fabric 
to eliminate non-deterministic transactions and increase the overall performance of the system. Further, this architecture helps Hyperledger Fabric to deliver high degree of confidentiality, resiliency, flexibility and scalability. Hyperledger fabric offers pluggable consensus, pluggable membership service provider, pluggable endorsement and validation policy, optional peer-to-peer gossip service and also helps to store ledger data in multiple formats. In Hyperledger fabric, the term consensus is defined as "the full circle verification of the correctness of a set of transactions comprising a block". The pluggable consensus option of Hyperledger Fabric allows it to fit different use cases and trust models in an efficient manner. The members of Hyperledger Fabric enroll through Membership Service Provider (MSP). The MSP is a component that offers an abstraction of all cryptographic mechanism and protocols behind issuing and validating certificates, and user authentication[8][9].

Fabric calls smart contracts as "chaincode" in which the business logic of the application is deployed. These smart contracts are run within container environment for the purpose of isolation. Hyperledger Fabric supports smart contracts to be written using general-purpose programming languages such as Java, Go, and Node.js which helps the developer to write applications easily and quickly.

The other distinguishing feature of Hyperledger Fabric is it provides confidentiality through its channel architecture. A channel is established between the subset of participants allowing only these subsets of participants to have visibility over a particular set of transactions. The privacy and confidentiality of transactions in Fabric network is preserved by allowing only the nodes that participate in the channel to access the chaincode and transaction data. The ledger subsystem supported by Hyperledger Fabric comprises two components namely World state and the transaction log. The world state is the database of the ledger which describes the current state of the ledger. The transaction $\log$ records all transactions that have resulted in the current value of the world state. It could be called as the update history for the world state.

Peers in the Hyperledger Fabric are assumed different roles and based on the roles assigned to them, they are called by different names.

1. Endorsing peer - Every peer with smart contracts installed are called endorsing peers.

2. Ordering peer - it is a peer that receive transactions from clients and order them into blocks.

3. Leader Peer - it is the peer that takes responsibility of collecting ordered transactions from the orderer and distributing them to the various committing peers.

4. Committing Peer - A peer that receives block of transactions and validate them before they are committed to the ledger.

5. Anchor peer - helps the peers to communicate with peers present in other organization.

This paper mainly focuses on drug supply chain along with inventory management at each stakeholder's point using Hyperledger Fabric Blockchain platform. First, the authors designed a drug supply chain system that stores the details about the drugs manufactured, demand from the wholesaler, distributor and pharmacies, supply of drugs to these demand points. The Hyperledger Fabric blockchain platform is used for this work, since it is an open-source permissioned blockchain designed for business use cases and supports modular architecture and general-purpose programming languages. Further, Couch DB is deployed to store large amount of transactions that are made between the stakeholders. Finally, the system is checked and the performance of the system is measured using the Benchmarking tool Hyperledger Caliper.

The paper is structured as follows. Section 2 elaborates the related work on applications of Blockchain in supply chain field, health care area and other related works. Section 3 gives an insight details about the proposed system architecture. Section 4 gives details about the system implementation. Section 5 elucidates execution results of the proposed system. Section 6 measures the performance in terms of success rate, transaction latency and throughput and evidences the result. Section 7 concludes the paper.

\section{Related Work}

\subsection{In Supply Chain}

[10] uses generic stochastic model to investigate the impact Blockchain Technology on the performance of supply chain. Concludes that leveraging Blockchain technology proves beneficial only to some types of goods and also recommends that Blockchain Technology should be adopted at an earlier stage and also at a higher degree.

[11] uses a mapping study method to explore and analyze the applications of Blockchain Technology in supply chain management. The study revealed that majority of the research focused on traceability, security and finance. Moreover, the study also concludes that real performance evaluation in terms of industrial context is lacking in almost all proposed frameworks and it needs to be taken into account by the researchers.

[12] investigates the applications and benefits of Blockchain Technology in Supply chain management. Developed a comprehensive framework to analyze the various applications of Blockchain Technology and identified five emerging use case clusters that could clearly extend the scope of Blockchain beyond tracking and tracing. 
A synthesis of the challenges that exist in global supply chain and trade operations is provided in [13]. It discusses how Blockchain could remediate the pain points of supply chain, how Blockchain could fulfill the needs of supply chain and logistics. It concludes that despite the benefits, there exists several legal and regulatory challenges in the wide adoption of this brainstorming technology in global supply chain market.

[14] Gives a report on digital Blockchain in supply chain which the organizations can make use of to understand how Blockchain technology is feasible for their applications and how to implement this booming technology for their applications. It also discusses how Supply chain is becoming complex over the years and the ability of Blockchain to remove these constraints.

A reference implementation named BLMS (Blockchain Based Logistics Monitoring System) based on Ethereum was programmed and tested to provide a solution for parcel tracking in a supply chain environment [15]. The system employs software components to record transaction entry for logistics operations and supply chain. The results prove that Blockchain is a promising technology that could increasingly streamline the supply chain environment by enabling sharing and access to product related information in real time.

A systematic analysis of 20-25 recent scholarly reviewed journals is done to know about innovations that Blockchain Technology could bring in the execution of supply chain and logistics management[16]. The study reveals that Blockchain Technology stands as the best option that could innovate today's business centers especially those with up-to-date machineries like online application of products and services.

[17] presents the results of a Blockchain Technology use case in particular, fresh food delivery designed using standard methodology. It evaluates the critical aspects of implementing Blockchain Technology and discusses how this groundbreaking technology helps in reducing logistics costs and in optimizing the operations of logistics. It also gives a quick depiction about the various issues such as scalability and costs of implementing this technology. Concludes that if Blockchain Technology is adopted in supply chain it could be a promising enhancement as well as, it provides benefits to all stakeholders involved in the system.

\subsection{In Health Care}

MedRec [18] uses blockchain technology to handle Electronic Medical Records (EMR) in which the patients are given a $\log$ of their medical history. The record is immutable, comprehensive, accessible and credible. Being source agnostic, MedRec is able to manage authenticity, confidentiality, data sharing and it provides incentives for medical researchers. This system aims to provide granularity, record flexibility and easy access of medical information between provider and treatment sites.

MediLedger, a project that developed a network which combines a Lookup directory accessed through a Blockchain with a permissioned messaging network in order to meet the demands of DSCSA (Drug Supply Chain Security Act). This allows only authorized companies to place their products in the Look-up directory as well as helps companies to request and respond to product identifier verification requests in a secure manner. Being an industry-owned permissioned blockchain network, it is able to solve the sensitive issues of data privacy. This decentralized network creates an open environment for pharmaceutical industry to overcome the limitations that the current method offers [19].

\subsection{Other works}

[20] presented a permissioned blockchain environment that provides trust and cost-efficient approach in academic publishing. It describes the benefits that academic publishing could get by the adoption of Blockchain Technology in terms of trust and collaboration between globally distributed participants without the need for centralized management.

A conceptual model for the fusion of Blockchain with Cloud computing is proposed in[21]. It comprises Blockchain over Cloud (BoC), Cloud over Blockchain ( $\mathrm{CoB}$ ) and Mixed Blockchain Cloud (MBC) deployment models and highlights the potential benefits of these fusion. The discussions were focused on secure data transfer and privacy issues associated with it. It also proposed a three layer model to reengineer cloud data centers using Blockchain Technology.

A blockchain based data logging and integrity management system for cloud forensics to prove the integrity of evidence collection and storage in the cloud environment is proposed in [22] and its performance is measured by comparing it with other blockchain based system. The proposed system outperforms other systems in terms of transaction processing and it guarantees data integrity.

In [23], a framework based on Blockchain and QR(Quick Response) Code is proposed to provide drug safety and manufacturer authenticity. The proposed medical storage makes use of permissioned private blockchain based on PKI and digital signatures. It discusses about how counterfeit drugs could be traced using the proposed blockchain methodology. It also proves that this methodology prevents replay and man-in-themiddle attack.

[24] Describes how blockchain could make a substantial difference to the current pharmaceutical supply chain model. It enables barcodes to be scanned and recorded on the Blockchain ledger at every stakeholder 
point. The record that is stored on the ledger helps to create an audit trial of the drug journey. It also allows the drug to be tracked from the time the drug is manufactured to the moment the patient receives the drug. The authors also enlighten the advantages of adopting blockchain technology in pharmaceutical supply chain. Further, in future, biometric measures could be used to record the dispenser and pharmacist details which could also be stored in the ledger for tracking purpose.

In [25], Gcoin Blockchain is used to create transparent drug transaction data. The double spending prevention mechanism given by the consortium Proof-of-Work approach of Gcoin helps to alleviate the counterfeit drug problem. In addition, the regulation model used in this work is surveillance net model which differs from the usual inspection and examination model. The surveillance net model allows every unit in the drug supply chain to participate simultaneously, helps to prevent counterfeit drugs, help to track and trace drugs without going into factories, warehouses or pharmacies.

Hyperledger Fabric based drug supply chain to manage integrity is proposed in [26]. The unique feature of Hyperledger Fabric system allows only valid participants to participate in the supply chain and make transactions. This system uses Proof-of-Concept which helps to keep track of drug records in a decentralized way, helps to achieve transparency, security and privacy. The performance of the system is tested by carrying out a number of experiments and is analyzed in terms of transaction response time, throughput, latency and resource utilization using the Benchmarking tool called Hyperledger Caliper. The paper concludes that using Blockchain Technology increases the performance of the system in terms of throughput and minimizes latency with less utilization of resources.

A quantitative analysis on leveraging Blockchain in the entire drug supply chain of India is proposed in [27]. The Blockchain database which is private and permissioned maintained by the Department of Pharmaceuticals is used as the distributed drug inventory which helps to maintain transactional records of supply chain. This helps to trace and track drugs at any level from the extraction unit till it reaches the patient. This work concludes that the journey of drug becomes more secure and streamlined through the use of potential Blockchain Technology.

DrugLedger: A blockchain system for drug regulation and drug tracing efficiently stores records and guarantees sustainable service delivery by reconstructing the whole service architecture. This system is more resilient than traditional systems and it provides data privacy and authentication. Drugledger tackles the various problems of package, and makes use of Expiration date of drugs to efficiently prune Blockchain storage [28].

A blockchain based e-prescription system that utilizes cryptocurrency principles is proposed in [29]. The authors in this work investigated the requirements of this system to run on a Blockchain network. The concept of mint is used as the fundamental concept of this proposed system. Rxcoin, a transferable currency on Ethereum

Blockchain is utilized to create a database of prescription data on blockchain that ensures integrity. This work also enlightens that the proposed e-prescription system could be a viable solution for combating opioid crisis. The impact of Blockchain technology in agriculture and food supply chain is examined in[30]. It demonstrates the current projects, initiatives and the various challenges associated with these projects. The authors elaborate that blockchain technology establish a proven and trusted environment for many projects and initiatives. The findings of the study reveal that Blockchain stands as a promising technology towards transparent food supply chain but still there exists many barriers and challenges that need to be solved before adopting this technology.

[31] identifies that the transparent nature of Blockchain technology when adopted in the supply chain furnishes the ability to secure favorable financing transactions. In this work, the authors develop b_verify, that utilizes Bitcoin to provide transparent supply chain both at scale and at lower cost. The analysis of this work demonstrates what types of firms or supply chain would benefit from the adoption of this Blockchain Technology. Finally, it concludes that Blockchain technology provides an efficient way to alleviate the problems of financing operations in small and medium sized enterprises (SME) by furnishing input transactions verifiability in supply chains.

BRUSCHETTA a blockchain based application for the traceability and the certification of Extra Virgin Olive Oil (EVOO) supply chain[32]. It provides a tamper proof record of the product from the point of plantation till it reaches the shop. This blockchain based application utilizes Internet of Things (IoT) to interconnect sensors used in EVOO quality control. From the results obtained, a mechanism for dynamic autotuning of BRUSCHETTA is proposed to optimize its performance in case of high loads. It concludes that since the transaction arrival rate vary over time, it is best to adopt dynamic configurable Blockchain instead of static configuration of Fabric Blockchain.

\section{Proposed System Architecture}

\subsection{Blockchain based Pharmaceutical Supply Chain}

Figure 2 illustrates the proposed Drug supply chain based on blockchain. It involves the Regulatory Authority, Manufacturer, Wholesaler, Retailer, Pharmacist and the Consumer. Every participant in the network updates, 
verifies and manages the supply chain data using the smart contracts deployed within it. Smart contracts are computer programs that define the roles and responsibilities of every participant in the network, the relationship among the participants in the network. It facilitates every participant in the network to interact with the distributed ledger. It also helps every participant in endorsing the transaction proposal and updating the ledger. The smart contracts receive the transaction request, execute the request and send the response back to the client. In the meanwhile, it queries the ledger, updates the ledger by appending information about the transaction. The working of smart contract is illustrated in Figure 3.

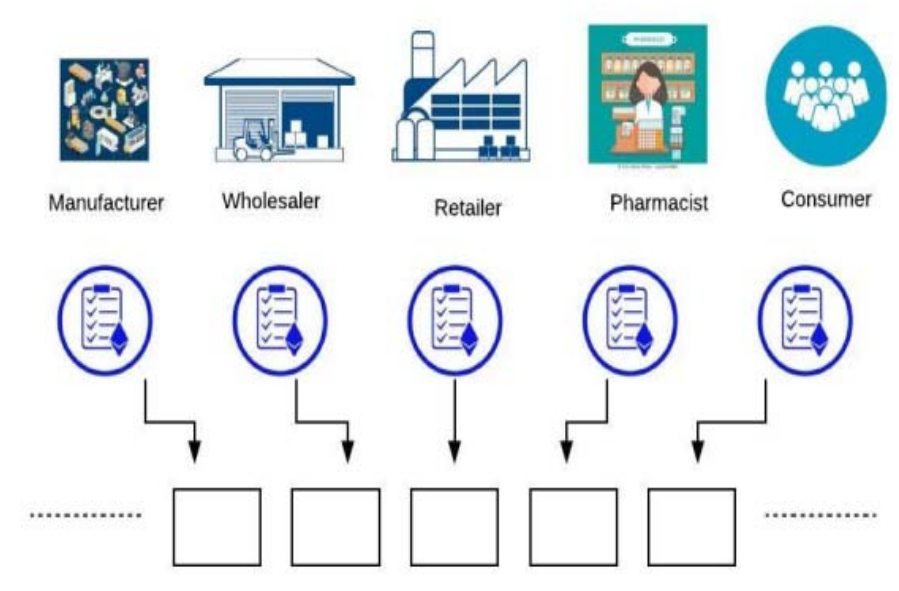

Figure.2 Proposed Drug Supply Chain System using Blockchain

The feature that makes the proposed system unique is that it is designed using a permissioned consortium network called Hyperledger Fabric. It allows only the authenticated participants to participate in the network and make transactions. The authenticity of the participants is verified using the certificate issued by the certificate authority.

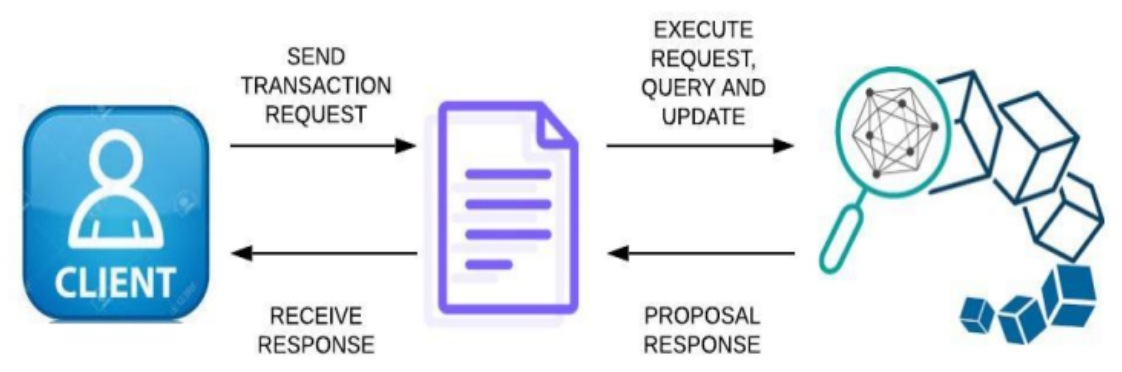

Figure.3 Working of Smart Contract

\subsection{Role of Participants}

The regulatory authority is responsible for setting up of the secure network by allowing only trusted parties to join and access the network while giving view-only permission to others in the network. The regulatory authority acts as the certificate authority and issues identity certificate to valid participants in the network.

The manufacturer is responsible for initiating the supply chain. After enrolling in the network, the manufacturer purchases the raw materials and manufactures the drug according to the formulary. He then enters the details of the manufactured drug product into the Blockchain which will be subsequently tracked by the participants in the supply chain network. The consistency of the information entered by the manufacturer is checked and endorsed by the regulatory authority. The manufacturer then sells the drug product according to the needs of the wholesalers.

The wholesaler sends the drug request to the manufacturer specifying the name and quantity of drugs needed. The manufacturer satisfies the request by sending the requested drug product and updates the information in the Blockchain. The wholesaler on receiving the items first verifies genuineness of the product using the information in the blockchain and then accepts and pays for the product. If the drug products received is not found to be genuine, then he would not accept the product and the payment would not be done. All these transactions are updated in the Blockchain.

The retailer receives the drug he needs, from the wholesaler and verifies the drug products he received are genuine using the information in the Blockchain. If the information is found to be correct, then the product is 
accepted and the payment is done. Otherwise, the product is discarded. This information is then updated in the Blockchain.

The pharmacist receives the product either from the manufacturer directly or from the retailer. He then verifies the integrity of the product received using the Blockchain information and updates the ledger.

The consumer purchases the drug prescribed by the doctor from the pharmacy. He can trace back the path of the drug using its identity. If the consumer finds any mismatch in the information about the drug, then the drug could be identified as a counterfeit one and the same could be reported to the regulatory authority. It is illustrated in Figure 4.

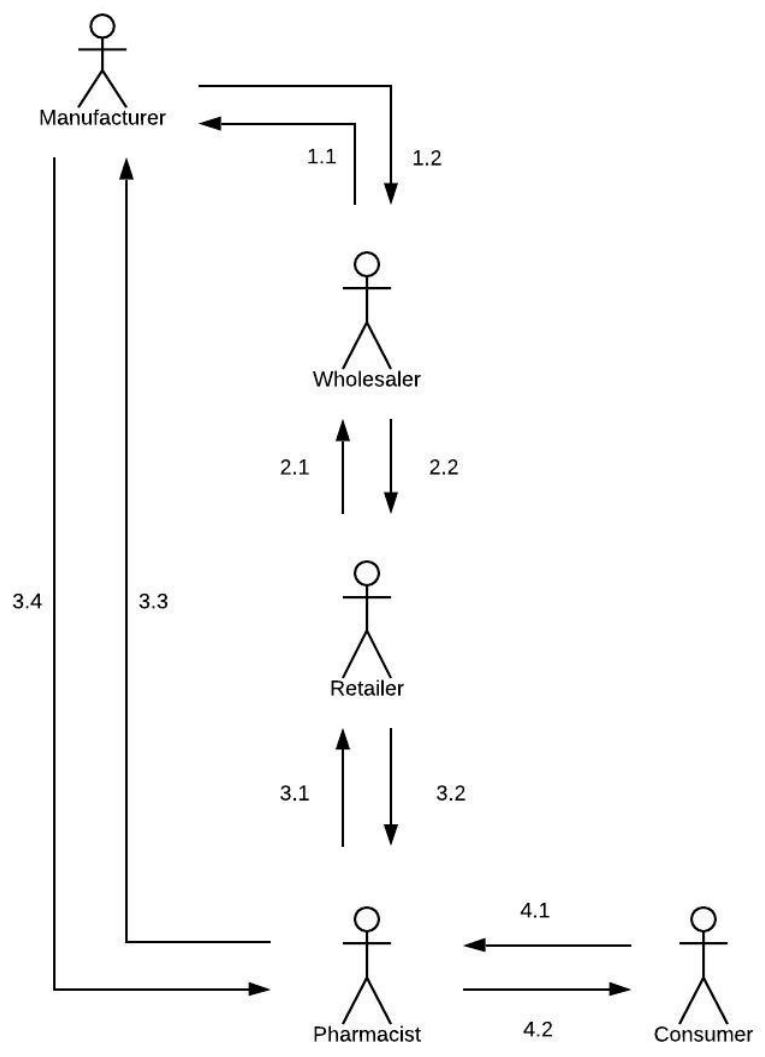

Figure.4 Role of Participants in the Drug Supply chain system

\subsection{Drug Movement}

In the supply chain, the movement of drugs starts from the manufacturing point and it changes hands till it reaches the end-user. This distribution chain involves a number of participants including the manufacturer, wholesaler, retailer and the pharmacist. The drug being manufactured has certain properties associated with it such as the drug name, drug form, dosage, manufacturing date, expiry date and manufacturer name. In addition, in this proposed system the drug has certain other properties such as drug id, drug owner, drug location, certificate number and temperature. When the drug is manufactured, it is given a unique id, name, form, dosage, manufacturing date, expiry date. These properties of the drug do not change until it reaches the end-user. The information such as drug owner, certificate number, drug location changes as it changes hands. When it is manufactured, the drug owner would be the manufacturer and the certificate number would be his registered certificate number and the drug location would be the place at which the drug is being manufactured. The location information of the drug could be given using the latitude and longitude position of the storage place of the drug. As the drug moves to the wholesaler, the owner information could be changed to the wholesaler id, the certificate number would be his certificate number and the location information is also updated. When the drug reaches the retailer, the certificate number of the retailer, retailer id and the location information is updated in the ledger. The same kind of update takes as the drug reaches the hands of pharmacist. The unique id of drugs helps the consumer to track the path back to its origin and he could identify the counterfeiting at any stage if there is any mismatch in the information provided by the blockchain. It is illustrated in Figure 5. 


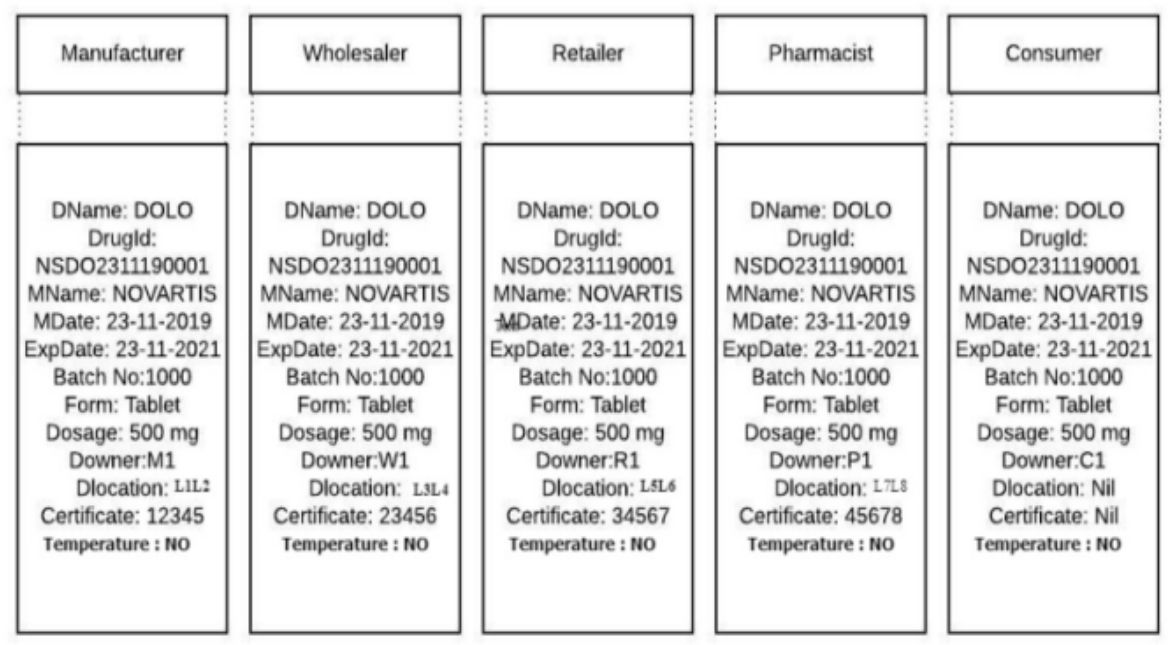

Figure.5 Drug Movement using Blockchain

\subsection{Flow of Transaction in the Proposed System}

During the movement of drugs in the supply chain, the nodes perform read and write transaction. After the nodes become members of the network by obtaining certificate from the certificate authority, they are allowed to perform the transactions according to the access control policy. The manufacturer, wholesaler, retailer and the pharmacist nodes are given both the read and write permission while the consumer node is given only the view permission. The communication between the nodes present in the network starts when a client node sends transaction proposal over the network. The endorsing nodes take the transaction proposal and execute the request using smart contracts. The smart contracts execute the transaction request and update the world state without updating the ledger. The proposal response is taken from the world state by the endorsing nodes and is signed with its certificate and return back to the client. The client node collects these responses and sends it to the ordering nodes. The ordering nodes are responsible for collecting these proposal responses from various clients and order into blocks. The blocks are then communicated to the committing nodes in the network which performs validation of the transaction response and commits the transaction by updating the ledger and the world state. The committing node could also generate an event about whether the transaction submitted by the client is successfully completed or not. The transaction flow for read and write transaction in the proposed drug supply chain is shown in Figure 6.

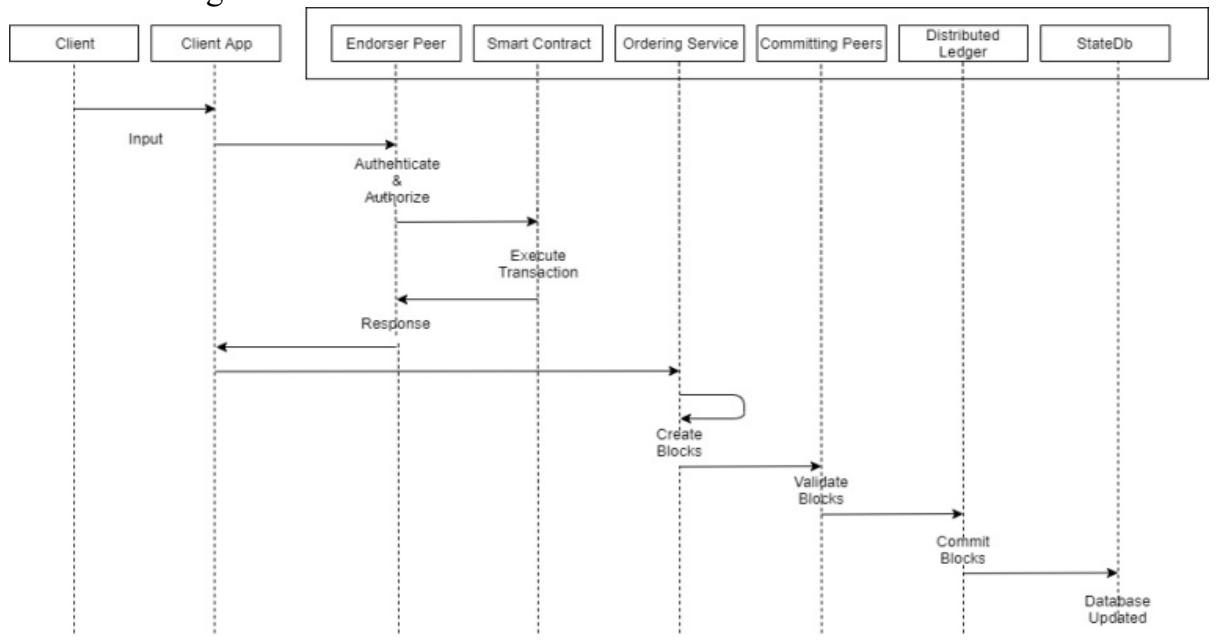

Figure.6 Transaction Flow for Read and Write operations in the proposed system

\section{System Implementation}

\subsection{Environmental Set up}

The proposed prototype makes use of Hyperledger Fabric, an open source permissioned Blockchain system to achieve the business logic behind the pharmaceutical drug supply chain system. The proposed system is implemented in Ubuntu 18.04 operating system with 16 GB memory and the processor used is Intel Core i5processor. The docker version used for running the docker environment is version 18.09 and the docker- 
compose used is version 18.09. The development environment of the proposed Drug Supply Chain system using Hyperledger Fabric is described in Table 1.

\begin{tabular}{|l|l|}
\hline Component & Description \\
\hline Operating system & Ubuntu 18.04 \\
\hline CPU & i5 Core Processor \\
\hline Memory & 16 GB \\
\hline Browser & Chrome/Firefox \\
\hline Hyperledger Fabric & Version 1.4 .3 \\
\hline Docker Compose & Version 18.09 \\
\hline Docker Engine & Version 18.09 \\
\hline Programming Language & Node.js \\
\hline Node & Version 8.11 .3 \\
\hline IDE & Visual studio code \\
\hline
\end{tabular}

\subsection{Network Structure of the Proposed System}

The network structure of the proposed system contains four organizations namely Manufacturer, Wholesaler, Retailer and the Pharmacist. These four organizations are connected through channel C1 and the ledger L1 associated with the channel $\mathrm{C} 1$ is maintained in the peer node P1. Each of the organization has a client application A1, A2, A3, A4 through which they communicate with the network. There is also another private channel C2 between Manufacturer and the Pharmacist and the Ledger L2 is maintained in the Peer node P2. Peer P3 present in the network maintains both $\mathrm{L} 1$ and $\mathrm{L} 2$ and is connected to both $\mathrm{C} 1$ and $\mathrm{C} 2$. Channel configuration policy (CCP1) governs Channel $\mathrm{C} 1$ and (CCP2) govern $\mathrm{C} 2$. There is an ordering node $\mathrm{O} 1$ which orders the transactions from various organizations into blocks. Certificate Authority CA1, CA2, CA3, CA4 is responsible for issuing the certificate to the members of the network. Figure 7 demonstrates the network structure of the proposed system.

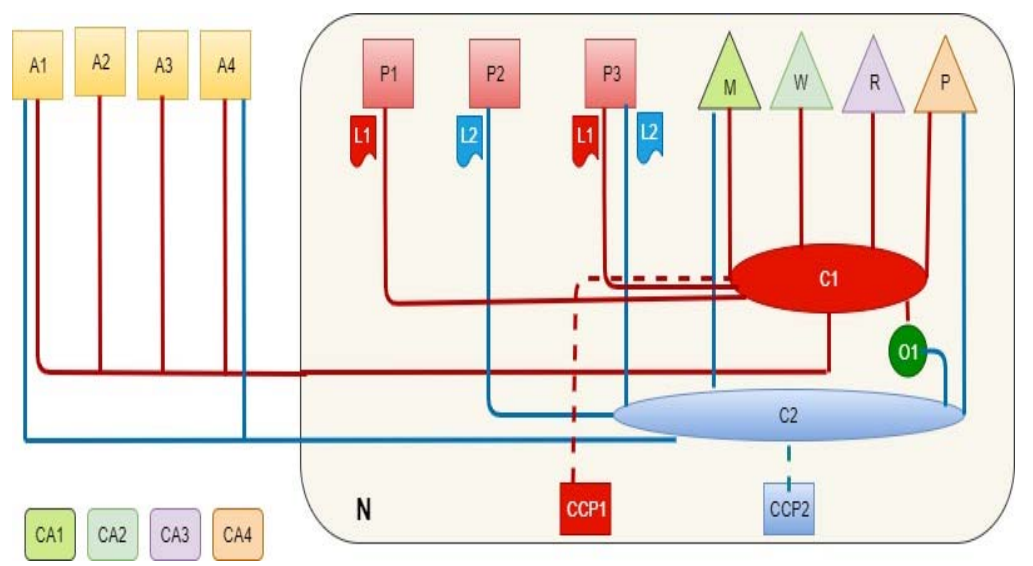

Figure.7 Proposed Network Structure

\section{Execution Results}

In this section, the execution of the proposed system is illustrated with the help of snapshots. The environmental set up of the proposed system is illustrated in Table 1. After successfully setting up the environment the chaincode is invoked. It is shown in Figure 8. Following this, admin and other members are registered in the network. Figure 9 shows the manufacturer login page. Using this, the manufacturer can login in to the network. The manufacturer after manufacturing the drugs can enter the details about the drug such as drug id, drug name, dosage, latitude and longitude value, manufacturing date, expiry date, manufacturer certificate number and temperature. Figure 10a and Figure 10b illustrates the manufacturer dashboard. After entering the details, when the manufacturer submits, a transaction id is created and the data is added to the database as shown in Figure 11. Figure 12 demonstrates the detailed database about the drugs manufactured by the manufacturer. This database could help the manufacturer to manage inventory. The manufacturer on viewing the database could come to know about the amount of drugs manufactured, expiry date of the drugs. Based on this database the manufacturer is able to sell drugs to the wholesalers and others in the network. 


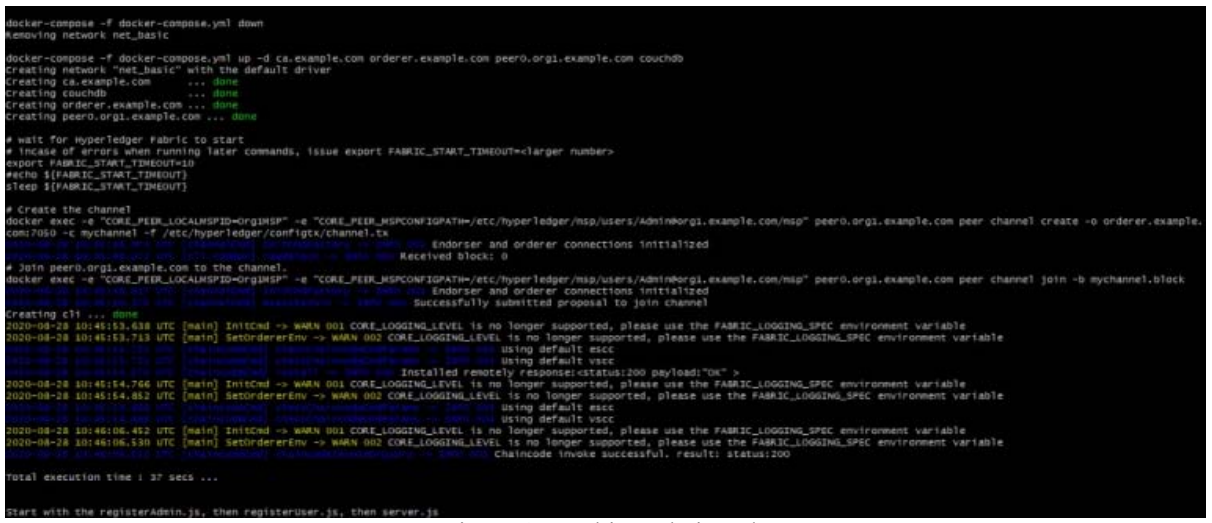

Figure.8 Invoking Chaincode

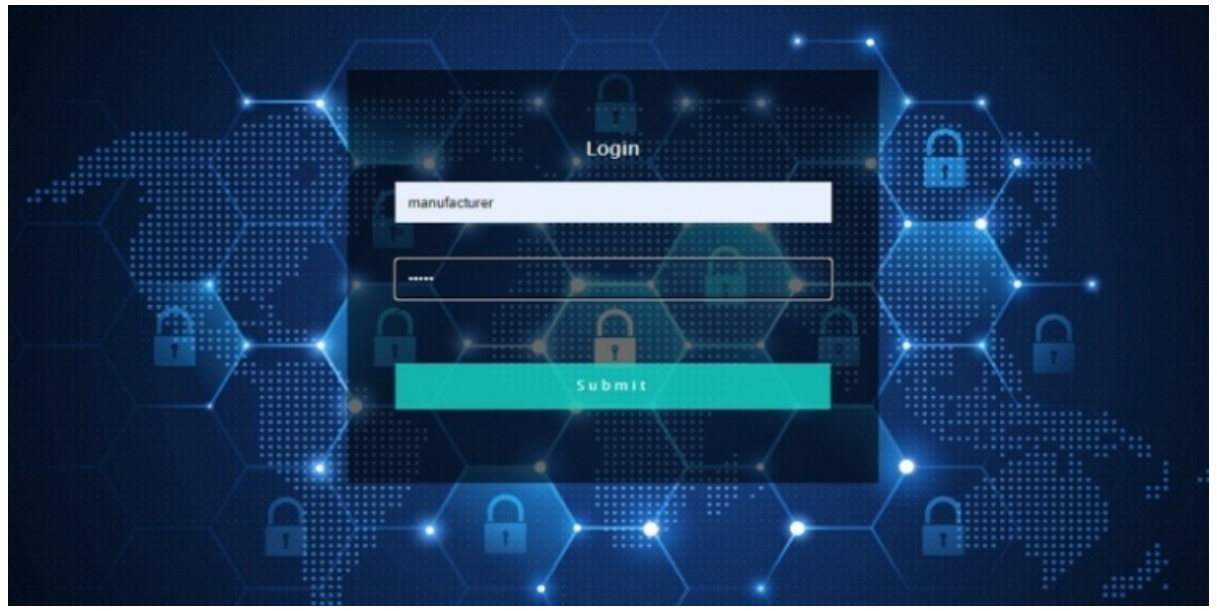

Figure.9 Manufacturer Login page

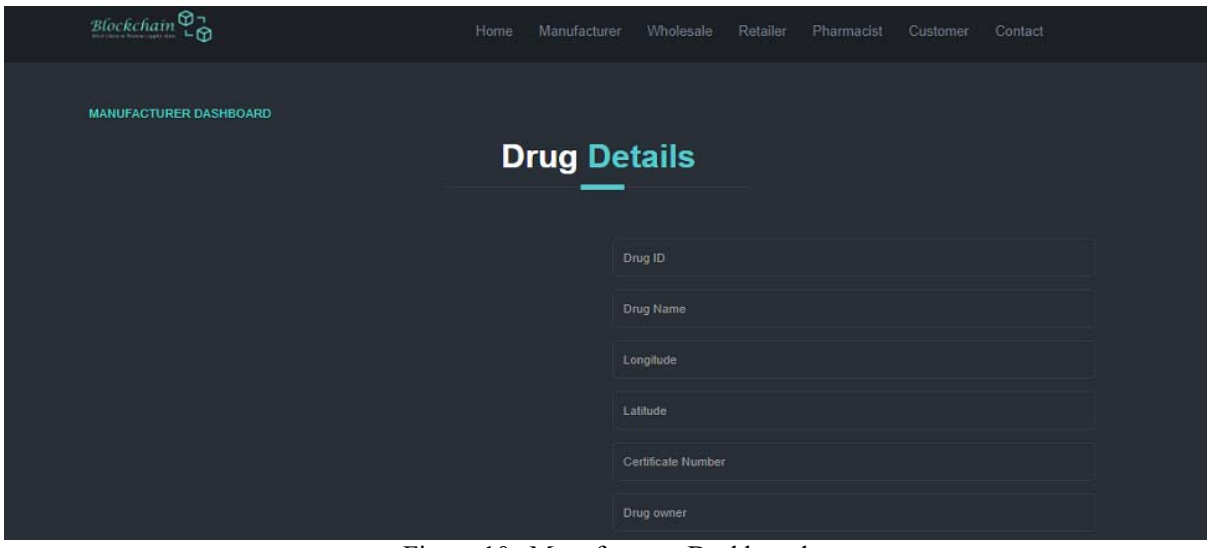

Figure.10a Manufacturer Dashboard

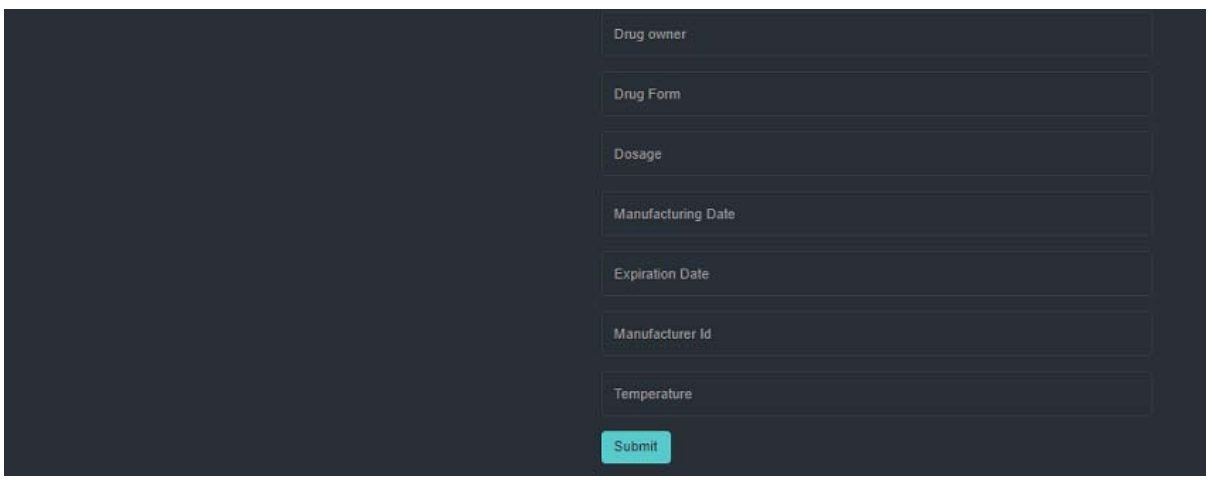

Figure.10b Manufacturer Dashboard 


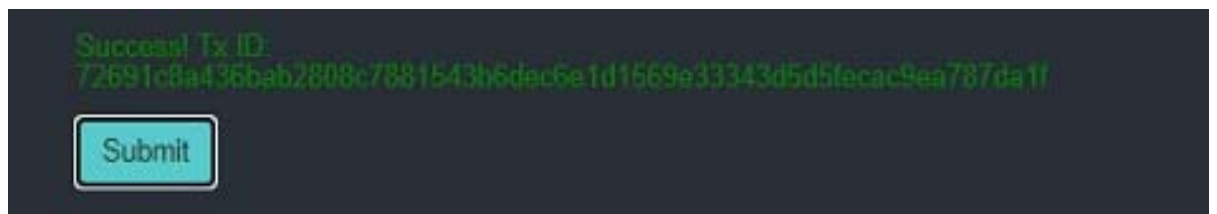

Figure.11 Transaction id created

\begin{tabular}{|c|c|c|c|c|c|c|c|c|c|c|}
\hline Shoofichain & & & & Hone & Manutactare & Wholetare & Retenter & Fharmacen & Costemer & Cotast \\
\hline Deas & 4 & covers & Molp Restoy & $14 \times 241256$ & $0 \mathrm{sm}$ & $\cos s$ & averapent & 200102023 & ASOMS & no \\
\hline Domentur & 5 & SHOS6 & Suctit Meres & $180 \mathrm{sss}, 14256$ & $10 \mathrm{mog}$ & Cost & 27rayzo16 & 1200002020 & 0003180 & no \\
\hline sikm & 6 & runer & Sutir Meres & $19 x a$ iscos & $100 \mathrm{mg}$ & coses & estrewens & $2 x 0402024$ & Gtकree & no \\
\hline Ceconom & 7 & Kusreas & $\begin{array}{l}\text { Oowh Bunter } \\
\text { costs }\end{array}$ & 1622413.256 & or forto & is & obecesst & 01002023 & corum & no \\
\hline Mosta & $\bullet$ & MESERE & $\begin{array}{l}\text { Denh enonter } \\
\text { Ouges }\end{array}$ & 15256.158253 & $25 \mathrm{mo}$ & tos & atramasoir & 200002024 & Hrats's & No \\
\hline Salmer & 9 & seners & Ranurenai Peat & $20 \operatorname{sen} 11 \times 6$ & $100 \mathrm{mog}$ & coss & nseppors & zenerases & reserces & no \\
\hline Proper & 10 & Pmisfef & Ramartad Prow & 10256.125297 & $0.5 \mathrm{mos}$ & Tho & o6seprovit & revenses & romeses & no \\
\hline Aletrompich & 1001 & NखाT & Weylevisen & $14254199 \times 9$ & $250 \mathrm{mog}$ & Tot & zenapors & 145002024 & nosieus & no \\
\hline freson & 1002 & foctos & Oarcy Pathp & $1135,128+155$ & 1000 & $c_{\infty}$ & THencong & 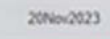 & FERCA & no \\
\hline $0=0$ & 1002 & प्रखर्या & maversen & 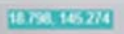 & 1000 & $\infty$ & सात्लग & xaras & 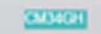 & $\bar{\Sigma}$ \\
\hline
\end{tabular}

Figure.12 Drug database

Similarly Figure 13 illustrates the wholesaler login page. Wholesaler after receiving the drugs from the manufacturer could check for the authenticity of the drug using the drug database. If he found that the drug comes from proper source he then accepts the drug and would change the drug holder name, drug holder certificate number, and latitude and longitude value of the drugs. Figure 14 illustrates the Wholesaler dashboard. After successfully changing the drug holder detail, a new transaction id is created as shown in Figure 15.

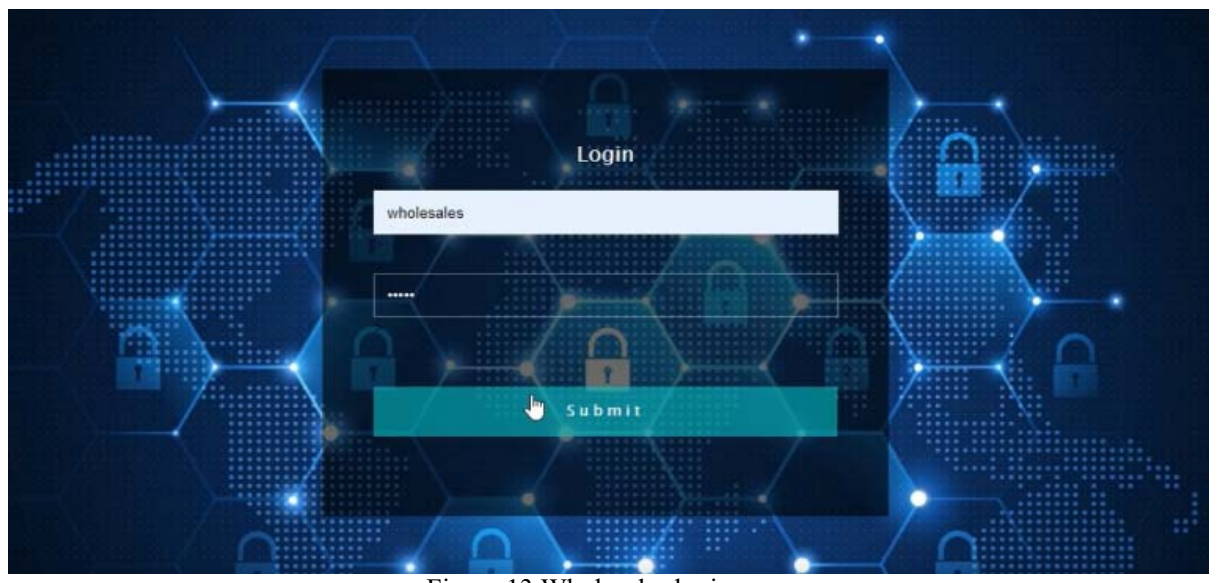

Figure.13 Wholesaler login page

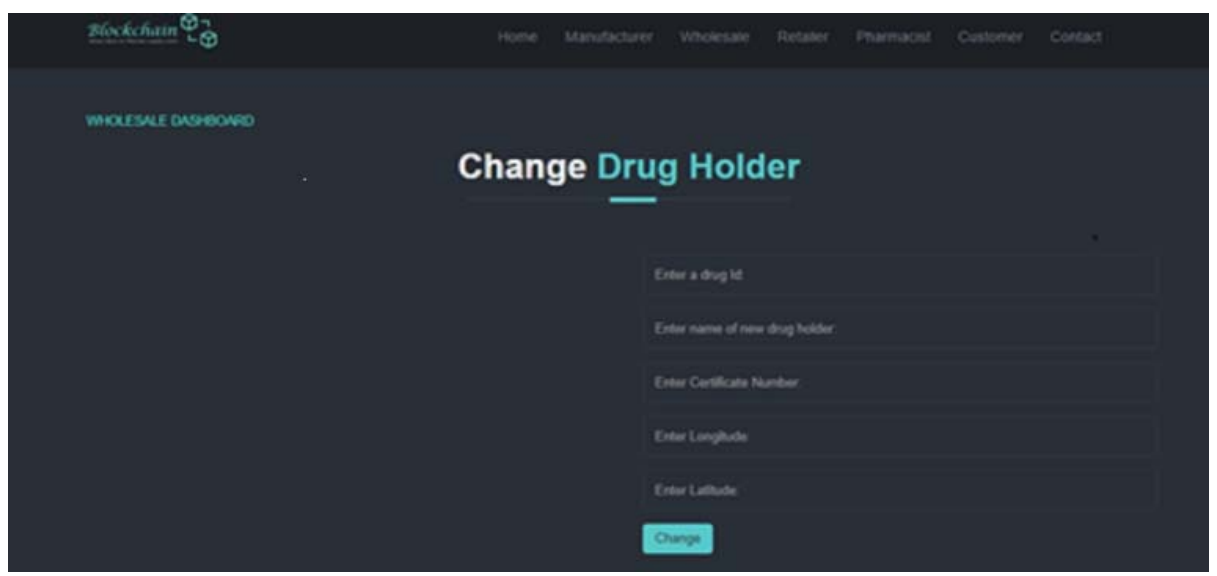

Figure.14 Wholesaler dashboard 


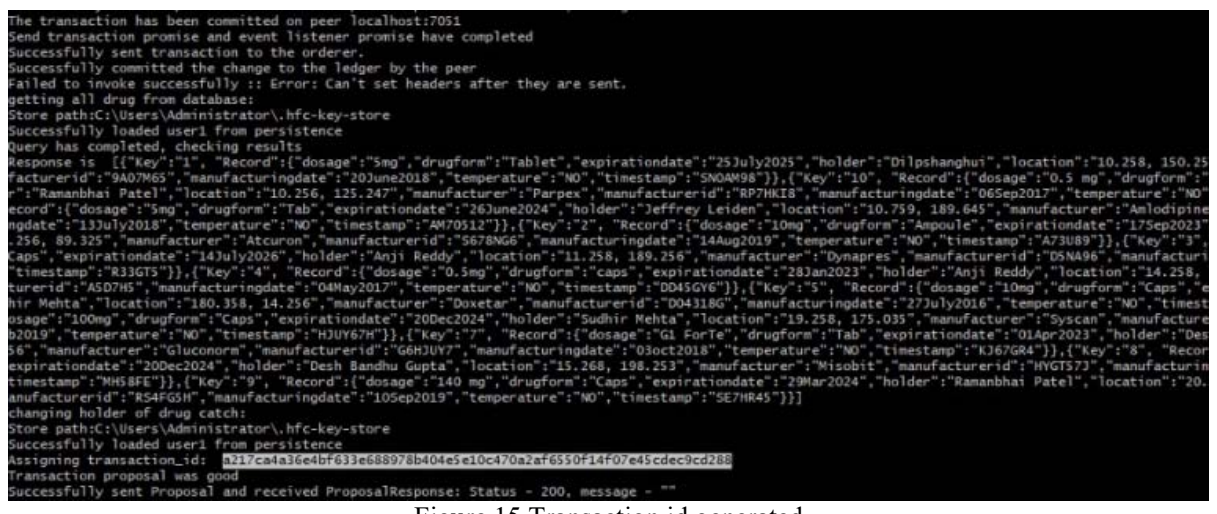

Figure.15 Transaction id generated

Figure 16, 17, 18 and 19 shows the retailer login page, retailer dashboard, pharmacist login page and pharmacist dashboard respectively. The retailer after logging in enters his dashboard. On receiving the drugs from the manufacturer or wholesaler, the retailer is able to check for the authenticity of the drugs and could change certain attributes of the drug such as drug id, drug holder name, certificate number of the drug holder, and latitude and longitude values of the drugs. Similarly Pharmacist on receiving the required drugs login into the network and update certain properties of the drug through his dashboard.

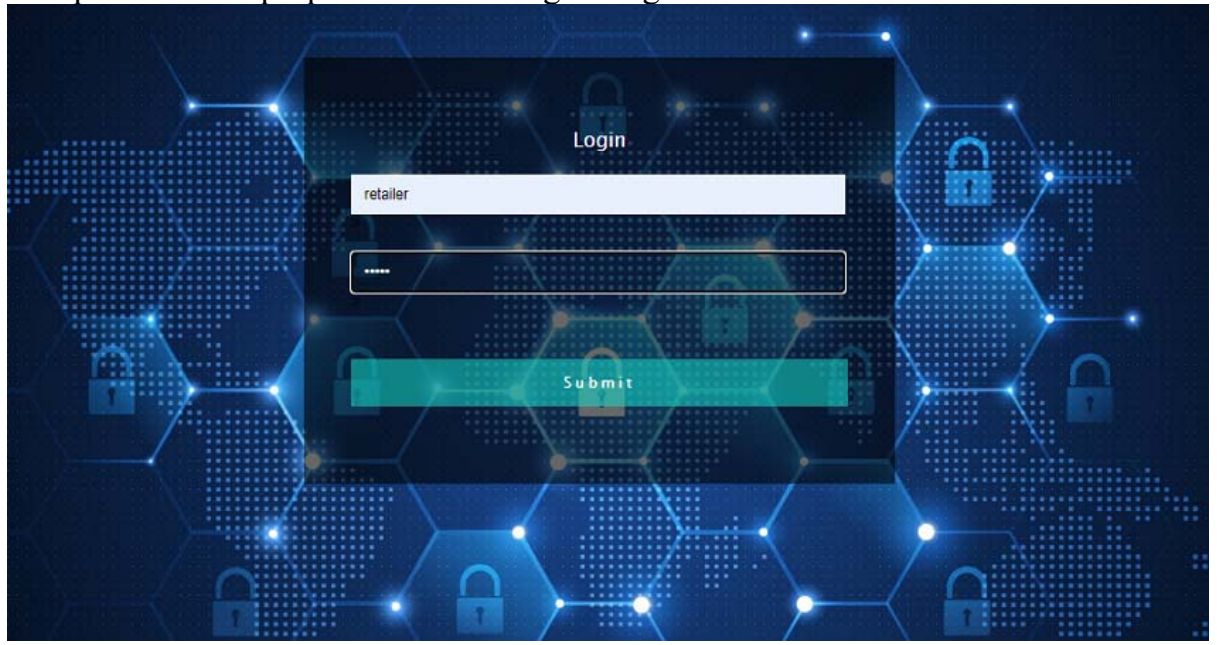

Figure.16 Retailer Login page

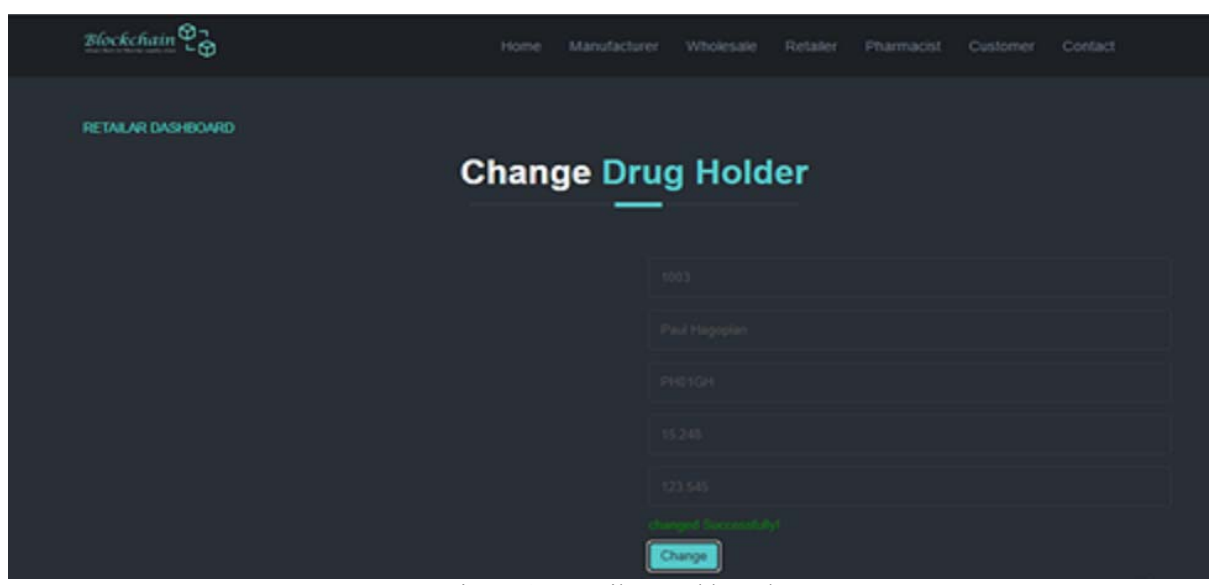

Figure.17 Retailer Dashboard 


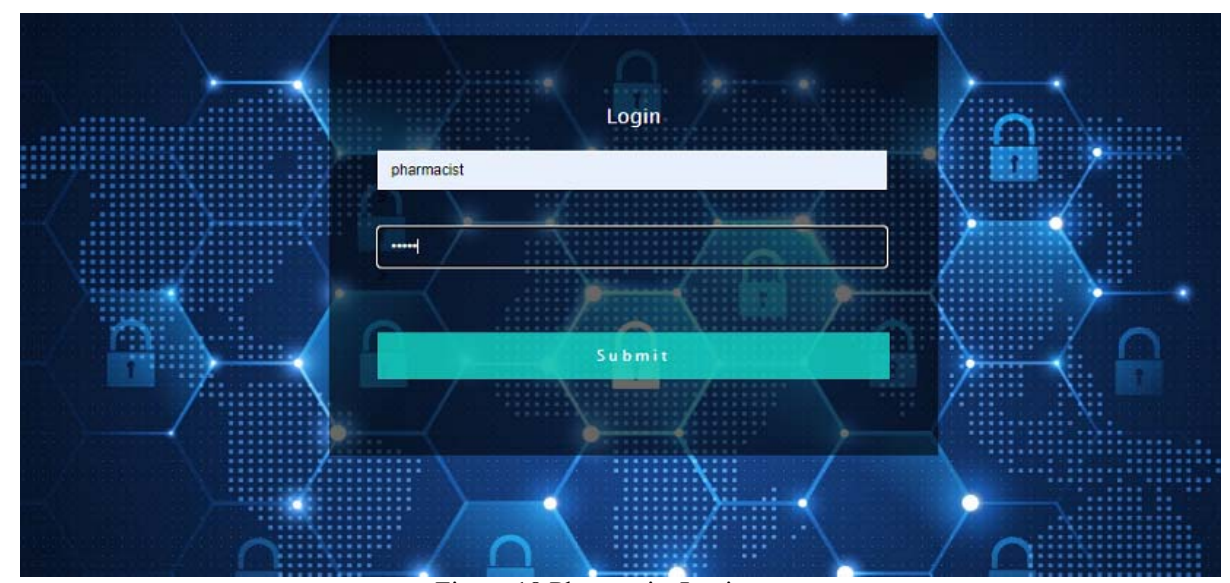

Figure.18 Pharmacist Login page

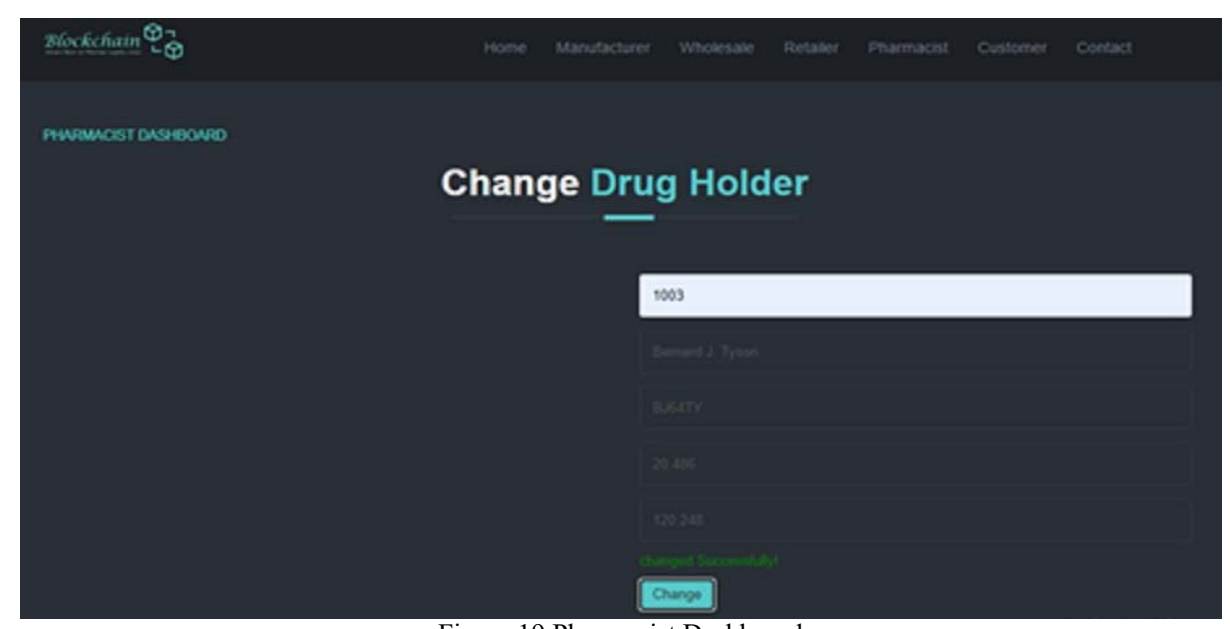

Figure.19 Pharmacist Dashboard

The customer who purchases the drug can check the authenticity of the drug using drug id. He can trace the path of the drug from the manufacturer till it reaches him. The customer will be provided with the details about the drug from the point of manufacturing. The customer does not have the right to update the details of the drug. He can only read the drug details. Figure 20 illustrates the customer dashboard. Figure 21 illustrates the path of the drug from the manufacturer to the pharmacist.

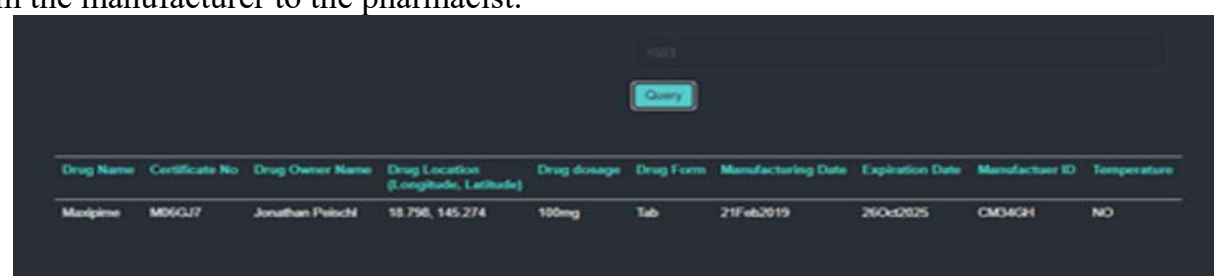

Figure.20 Customer Dashboard 


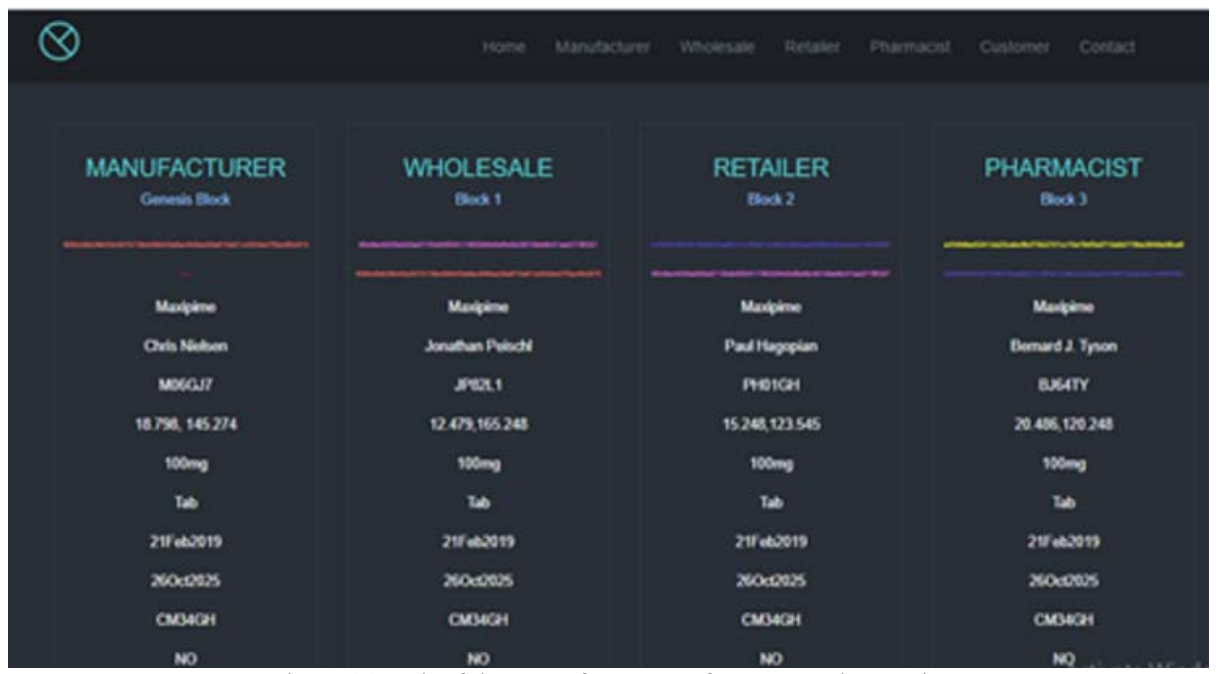

Figure.21 Path of the Drug from Manufacturer to Pharmacist

\section{Performance Measurement}

It is one of the most concerned features to measure the performance of a blockchain solution. Hyperledger Caliper is a blockchain benchmark tool that helps to measure the performance of a blockchain implementation. Currently, it supports Hyperledger Besu, Hyperledger Fabric, Hyperledger Iroha, Hyperledger Burrow Hyperledger sawtooth and Ethereum[33]. The reports produced by the Hyperledger Caliper contain a number of performance indicators such as Transaction latency, Transaction per second, success rate. The environmental set up to measure the performance is illustrated in Table 2. After successfully setting up the environment, the performance is measured by running caliper and the results are produced against the following metric.

1. Success Rate

2. Transaction Latency

3. Throughput

\begin{tabular}{|l|l|}
\hline S.No & Component \\
\hline 1 & Node-gyp \\
\hline 2 & Python \\
\hline 3 & Node.js v8.11.4 \\
\hline 4 & Docker engine v18.06.1-ce \\
\hline 5 & Caliper v0.2.0 \\
\hline
\end{tabular}

Table.2 Environmental set up to measure performance

\subsection{Success Rate}

It is the measure of percentage of successful and failed transactions for a test cycle. We measured the success rate using 5 groups of users such as 10 users at the first round, 30 users at the second round, 50 users at the third round, 70 users at the fourth round and finally 100 users at the last round. Figure 22 shows the success rate at all the five rounds. It is found that when the number of users is 10 and 30 the success rate is 100 percentage. When the number of users sending request at the same time is increased to 50 , the success rate is found to be 99 percentage and it falls to 97.5 percentage when the number of users is increased to 70 . It is also observed that when there are 100 users in the system the success rate is about 96 percentage. It can be concluded that the rate of success begins to decrease when the number of users gets increased.

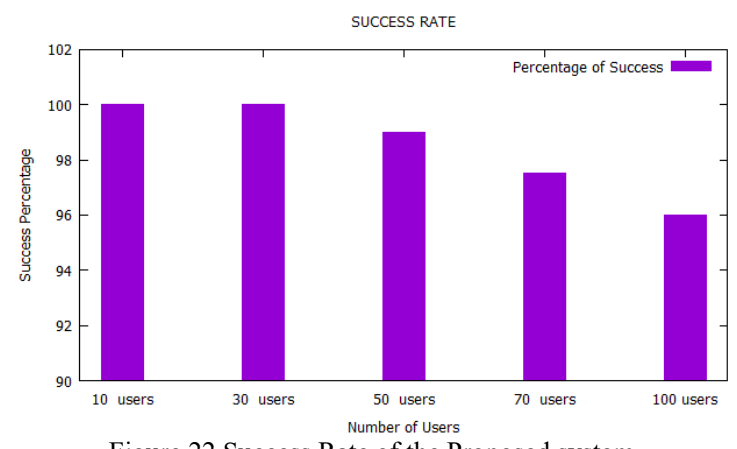

Figure.22 Success Rate of the Proposed system 


\subsection{Transaction Latency}

It is defined as the measure of time from the point of submitting a transaction till it is available across the network. The transaction latency for the proposed system is measured by invoking the transaction using 10,30 , 50,70 and 100 users. The transaction latency for invoke is higher since it involves endorsement function. Figure 23 demonstrates the minimum, average and maximum latency at each round using different user groups. It is found that minimum and average latency does not have much difference in case of 10 and 30 users. When the number of users is increased to 50 the latency begins to increase and in case of 70 and 100 users the maximum latency is found to be very high. It is concluded that the transaction latency is high when the numbers of users in the system gets increased.

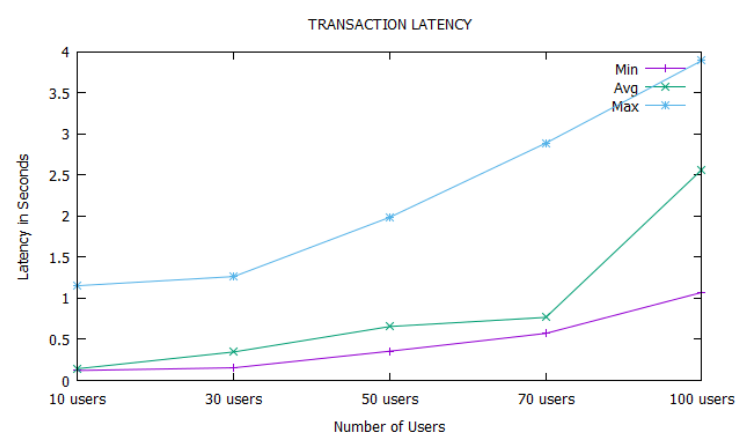

Figure.23 Minimum, Average and Maximum Latency for the Invoke transaction of proposed system

\subsection{Throughput}

It measures the rate of flow of all transactions through the system. It is measured in Transactions per second. In the proposed system, this metric is evaluated using five groups of users and is illustrated in Figure 24. The throughput is found to be constant upto 50 users in the system. When the number of users is increased from 50, the throughput starts to decline and it is found to be very low when there are 100 users in the system. It could be concluded that the proposed system shows a good throughput of an average of 95 TPS for upto 50 concurrent users in the system.

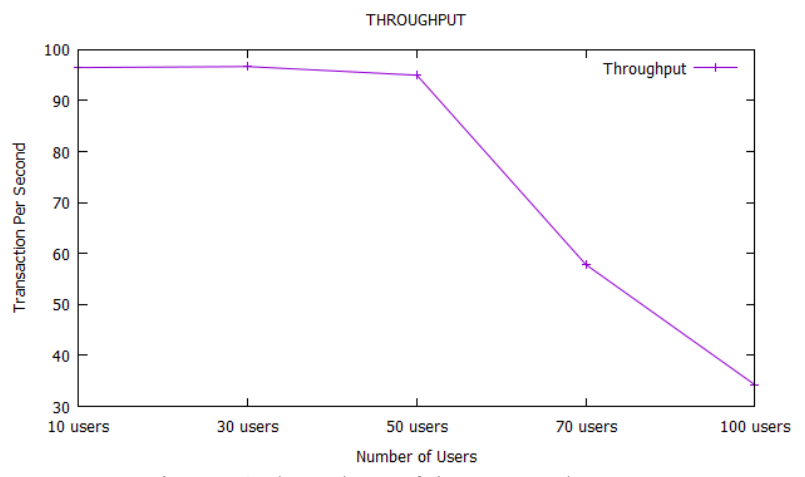

Figure.24 Throughput of the Proposed System

\section{Conclusion and Future Work}

Blockchain, the revolutionary technology has the capacity to reshape the traditional supply chain system. This technology in particular could miraculously impact the drug supply chain management system. This paper described the pitfalls in current drug supply chain system and proposed a novel solution using hyperledger fabric blockchain. The proposed system could be called as the proof-of-concept application that helps to track drugs from the point of manufacturing till it reaches the consumer. In this system, the manufacturer, wholesaler, retailer and the pharmacist has the right to update records in the database whereas the consumer has the right only to view the records. The consumer is not allowed to perform any update operation. A web application has been developed in order to provide interaction between the blockchain platform and the users in the system. The proposed system is secure since only the registered members can have access to the system and the authenticity of the system is ensured with the help of digital certificates. Drug supply chain system proposed using Blockchain 2.0 promises the supply of drugs in a secure and accountable manner. The performance of the proposed system is measured using Hyperledger Caliper, a blockchain benchmark tool. The system undergoes a number of rounds of experiments with different user groups and the metrics such as success rate, transaction latency and transaction per second are measured. It is found that the success rate begin to decrease with an increase in the number of users, there is a decrease in number of transaction per second with the increase in 
number of users and there is a maximum latency in case of increased number of users. Further in future, the system could be designed to support cross-chain platform and improve throughput and success rate with an increase in the number of users in real-time.

\section{Funding}

This research did not receive any specific grant from funding agencies in the public, commercial or not-forprofit sectors.

\section{References}

[1] [1] W. Davies, "The escalating pharma counterfeit problem," 2018.

[2] [2] "1 in 10 medical products in developing countries is substandard or falsified." [Online]. Available: https://www.who.int/newsroom/detail/28-11-2017-1-in-10-medical-products-in-developing-countries-is-substandard-or-falsified. [Accessed: 13-Oct-2020].

[3] [3] "India's pharma industry expected to grow to $\$ 55$ bn by 2020." [Online]. Available: https://www.inoxpa.in/company/news/indias-pharma-industry-expected-to-grow-to-55-bn-by-2020. [Accessed: 13-Oct-2020].

[4] [4] D. Kapoor, “An Overview on Pharmaceutical Supply Chain: A Next Step towards Good Manufacturing Practice," Drug Des. Intellect. Prop. Int. J., vol. 1, no. 2, pp. 49-54, 2018, doi: 10.32474/ddipij.2018.01.000107.

[5] [5] "Pharmacy Inventory Management \& Removal Processes | Study.com." [Online]. Available: https://study.com/academy/lesson/pharmacy-inventory-management-removal-processes.html. [Accessed: 13-Oct-2020].

[6] [6] S. Nakamoto, "Bitcoin: A Peer-to-Peer Electronic Cash System."

[7] [7] P. U and N. Rajagopalan, "Pharmaceutical Cold Chain Using Blockchain 3.0," Int. J. Psychosoc. Rehabil., vol. 23, no. 1, pp. 202-209, Feb. 2019, doi: 10.37200/ijpr/v23i1/pr190230.

[8] [8] "Key Concepts — hyperledger-fabricdocs master documentation." [Online]. Available: https:/hyperledgerfabric.readthedocs.io/en/release-1.4/key_concepts.html. [Accessed: 13-Oct-2020].

[9] [9] "Architecture Reference — hyperledger-fabricdocs master documentation." [Online]. Available: https://hyperledgerfabric.readthedocs.io/en/release-1.4/architecture.html. [Accessed: 13-Oct-2020].

[10] [10]J. Chang, M. N. Katehakis, B. Melamed, and J. (Junmin) Shi, "Blockchain Design for Supply Chain Management," SSRN Electron. J., 2018, doi: 10.2139/ssrn.3295440.

[11] [11]Y. Tribis, A. El Bouchti, and H. Bouayad, "Supply chain management based on blockchain: A systematic mapping study," MATEC Web Conf., vol. 200, 2018, doi: 10.1051/matecconf/201820000020.

[12] [12]G. Blossey, J. Eisenhardt, and G. Hahn, "Blockchain Technology in Supply Chain Management: An Application Perspective," Proc. 52nd Hawaii Int. Conf. Syst. Sci., vol. 6, pp. 6885-6893, 2019, doi: 10.24251/hicss.2019.824.

[13] [13]Y. Chang, E. Iakovou, and W. Shi, "Blockchain in global supply chains and cross border trade: a critical synthesis of the state-ofthe-art, challenges and opportunities," Int. J. Prod. Res., pp. 1-18, 2019, doi: 10.1080/00207543.2019.1651946.

[14] [14]"Does blockchain hold the key to a new age of supply chain transparency and trust? How organizations have moved from blockchain hype to reality."

[15] [15]P. Helo and Y. Hao, "Blockchains in operations and supply chains - a review and reference implementation," Proc. Int. Conf. Comput. Ind. Eng. CIE, vol. 2018-Decem, no. July, 2018, doi: 10.1016/j.cie.2019.07.023.

[16] [16]M. Shamout, "Understanding blockchain innovation in supply chain and logisticsindustry," Int. J. Recent Technol. Eng., vol. 7, no. 6 , pp. $616-622,2019$.

[17] [17]G. Perboli, S. Musso, and M. Rosano, "Blockchain in Logistics and Supply Chain: A Lean Approach for Designing Real-World Use Cases," IEEE Access, vol. 6, pp. 62018-62028, 2018, doi: 10.1109/ACCESS.2018.2875782.

[18] [18]A. Azaria, A. Ekblaw, T. Vieira, and A. Lippman, "MedRec: Using blockchain for medical data access and permission management," Proc. - 2016 2nd Int. Conf. Open Big Data, OBD 2016, pp. 25-30, 2016, doi: 10.1109/OBD.2016.11.

[19] [19]"MediLedger - Blockchain solutions for Pharma companies.” [Online]. Available: https://www.mediledger.com/. [Accessed: 04Nov-2019].

[20] [20]P. Novotny et al., "Permissioned blockchain technologies for academic publishing," Inf. Serv. Use, vol. 38, no. 3, pp. 159-171, 2018, doi: 10.3233/ISU-180020.

[21] [21]K. Gai, K. K. R. Choo, and L. Zhu, "Blockchain-Enabled reengineering of cloud datacenters," IEEE Cloud Comput., vol. 5, no. 6, pp. 21-25, 2018, doi: 10.1109/MCC.2018.064181116.

[22] [22]J. H. Park, J. Y. Park, and E. N. Huh, "Block Chain Based Data Logging and Integrity Management System for Cloud Forensics,” pp. 149-159, 2017, doi: 10.5121/csit.2017.71112.

[23] [23]R. Kumar and R. Tripathi, "Traceability of counterfeit medicine supply chain through Blockchain," 2019 11th Int. Conf. Commun. Syst. Networks, COMSNETS 2019, vol. 2061, no. 1, pp. 568-570, 2019, doi: 10.1109/COMSNETS.2019.8711418.

[24] [24]“(No Title)." [Online]. Available: https://www.pwc.co.uk/healthcare/pdf/health-blockchain-supplychain-report v4.pdf. [Accessed: 13-Oct-2020].

[25] [25]J. H. Tseng, Y. C. Liao, B. Chong, and S. W. Liao, "Governance on the drug supply chain via gcoin blockchain," Int. J. Environ. Res. Public Health, vol. 15, no. 6, 2018, doi: 10.3390/ijerph15061055.

[26] [26]F. Jamil, L. Hang, K. H. Kim, and D. H. Kim, “A novel medical blockchain model for drug supply chain integrity management in a smart hospital," Electron., vol. 8, no. 5, pp. 1-32, 2019, doi: 10.3390/electronics8050505.

[27] [27]A. Kumar, D. Choudhary, M. S. Raju, D. K. Chaudhary, and R. K. Sagar, "Combating Counterfeit Drugs: A quantitative analysis on cracking down the fake drug industry by using Blockchain technology," 2019 9th Int. Conf. Cloud Comput. Data Sci. Eng., pp. 174-178, 2019, doi: 10.1109/confluence.2019.8776891.

[28] [28]K. Fan, Y. Ren, and Z. Yan, “on Blockchain,” 2018 IEEE Int. Conf. Internet Things IEEE Green Comput. Commun. IEEE Cyber, Phys. Soc. Comput. IEEE Smart Data, pp. 1349-1354, 2018, doi: 10.1109/Cybermatics.

[29] [29]C. Thatcher and S. Acharya, "Pharmaceutical uses of Blockchain Technology," Int. Symp. Adv. Networks Telecommun. Syst. ANTS, vol. 2018-Decem, pp. 1-6, 2019, doi: 10.1109/ANTS.2018.8710154.

[30] [30]A. Kamilaris, A. Fonts, and F. X. Prenafeta-Boldv́, "The rise of blockchain technology in agriculture and food supply chains," Trends Food Sci. Technol., vol. 91, pp. 640-652, 2019, doi: 10.1016/j.tifs.2019.07.034.

[31] [31]J. Chod, N. Trichakis, G. Tsoukalas, H. Aspegren, and M. Weber, "On the Financing Benefits of Supply Chain Transparency and Blockchain Adoption," pp. 1-35, 2019.

[32] [32]A. Arena and C. Vallati, "BRUSCHETTA : An IoT Blockchain-Based Framework for Certifying Extra Virgin Olive Oil Supply Chain,” 2019, doi: 10.1109/SMARTCOMP.2019.00049. 
[33] [33]"Hyperledger Caliper | Caliper is a blockchain performance benchmark framework, which allows users to test different blockchain solutions with predefined use cases, and get a set of performance test results." [Online]. Available: https://hyperledger.github.io/caliper/. [Accessed: 13-Oct-2020].

\section{Authors Profile}

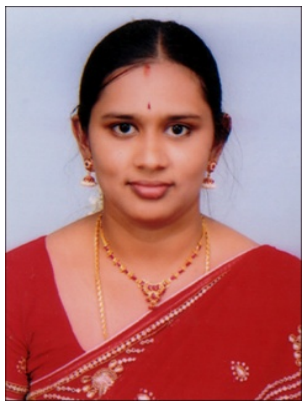

U. Padmavathi received her M.E degree in Computer Science \& Engineering from Annamalai University, Chidambaram, India in the year 2011. Currently, She is Pursing her $\mathrm{PhD}$ in National Institute of Technology Puducherry, Karaikal, India. Her research Interest include Blockchain, Networks and Security.

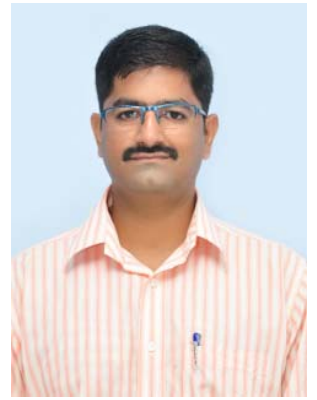

Narendran Rajagopalan completed his Ph.D from NIT Tiruchirappalli in 2013 and is currently serving as Assistant Professor and Head in the department of Computer Science and Engineering, National Institute of Technology Puducherry, India. His research interests include Networking, security and Quality of Service. 